\title{
Research and Implementation of RGB-LED Mixed Light Matching along with Music Playing
}

\author{
Zhang Hong, Gao Xiaoshuang, Mohanmmed Obadi, Zhao Ruirui, Tong Jinrui, \\ Tian Chunwei, Hu Zhiqiang \\ (School of Computer Science and Technology, \\ Harbin University of Science and Technology, \\ Post Box 073, Xuefu Road 52, Harbin 150080, China) \\ zh_e_mail@sina.com
}

\begin{abstract}
Synesthesia effect between music and color is a researching direction which is concerned much. The problem of mismatching between music playing and color had always existed in applications. On the basis of researching music and color, colors which are corresponding to various chords respectively are determined, and the frequency spectrum analysis on chords is performed. The real-time frequency domain transformation to time domain audio signal being played is carried out. The closest chord to audio signal being played can be identified via comparison between a chord spectrum and the spectrum of audio signal being played, and the color corresponding to the audio signal is determined. To improve the accuracy of recognition the method is investigated with which wavelet transform is adopted to de-noise processing of audio signal. Through tests on the audio experiment platform, color mixed effect test, optimization of the audio signal processing parameters, and processing time sequence scheming have been performed. A novel audio player is developed in application to realize the optimal RGB-LED mixed light which automatically presents matching color effect for arbitrary music being played. The research can reveal relationships between music and color from the perspective of frequency spectrum, promote the development of the color music, which has broad application prospects in urban landscape, stage lighting, and psychotherapy.
\end{abstract}

Keywords: Synesthesia, Audio playing, music chord, Spectrum Analysis, RGB-LED

\section{Introduction}

Relationships between music and color is various, people have been trying to explore inter-relations between sound and light color. For example, the voice frequency range, which is audible to the human ears, associates with the spectrum of visible light ribbons proportionally. Generally color-visual and music-audition interweaving act on human beings, which give some aesthetic and psychological enjoyment. This psychological phenomenon is called synesthesia[1,2]. It is a common phenomenon that synesthesia is human sensory perceptions[3]. Synesthesia is figuratively described as the ability that people "hear" colors and "see" the music[4]. Synesthesia phenomenon has a huge effect on sense and psychology, and provides a theoretical basis in the aspect of psychology to the development of color music, music visualization technology. 1

Explorations on relations between music and color have undergone a long period. But previous researches have either just concerned about the impact on human beings mentality caused by synesthesia co-acted by music and color, or just on electronic technology to develop music players, without much attention on studying the relationship between music and color. There are lots of music players, but among them, most have no

Zhang Hong: Corresponding author. 
light shining function at all, although some have it, music and light color have no relevance, music playing does not correspond to the color of light synchronously, even some just play with light shining following prescheduled color pattern. Music which possesses instantaneity and variety can be played in different ways and styles. It is difficult to determine a color to match a playing music in real time. There always exists mismatching between music playing and colors. In order to solve the problem of the mismatching to reach the effect that colors can be automatically corresponded to any music played arbitrarily, the primary work should be finished, which includes the mapping between standard chords and colors, recognition on spectrum of playing music, colors formed by mixed light from RGB-LED. Different from previous studies, main methods adopted in this paper are spectrum analysis and comparisons of frequency spectrums among music playing and music chords. The corresponding relationship between music chords and color has been investigated and the method of audio spectrum identity has been researched. The frequency spectrums of chord are got by using spectrum analysis. The frequency spectrum of music playing can be determined by Fast Fourier Transformation (FFT)[5]. The pattern of a moment music playing can be determined by comparing the spectrum of music playing and that of chords, and the most similar chord to the music playing is chosen, and then the color corresponding to the music is determined. By PWM driving circuit, RGB_LED light color can be controlled along with rhythms, pitch and color of music playing. This research can promote the development of music and color matching device, has broad application prospects in the urban landscape, stage lighting, and other aspects of psychotherapy.

\section{System Research Method}

There is some mapping relation between a music chord and a color according to musician studies. Due to the complexity of the music changes, it is impossible to determine a corresponding color just by its spectrum. The system research method in this paper includes three aspects. Firstly, on the basis of predecessors' studies on music, some standard music chords are chosen, and their audio signals are transformed from time domain into frequency domain. Secondly, audio signals of music playing are sampled in interval on an experiment platform, and their frequency spectrums are got by FFT transform in real time. Thirdly, a color correspondent to music playing at a moment is determined by comparing frequency spectrums between that of music playing and a chord.

\subsection{Music and Color}

Combination of music and color produces synesthesia, which causes neurological and psychological impacts on human consciousness, gives rise to a wonderful illusion, and a variety of feelings. Relations between music and color are multifaceted. Many people try to find links between audio and light waves. Historically it is the composer's works that makes people associate with colors, such as some say Mozart's music is blue, Chopin's music is green, Wagner's music is flashing different colors, and so on. Although these claims are general, it still shows that people often associate music with colors. The famous composer Rimsky Korsakov and pianist Scriabin, all thinks that tonal color is obvious, and proposed their specific ideas. Rimsky considers that $\mathrm{C}$ major is corresponding to the white, G major brown, gold, D major yellow, A major is rosy, E-flat sapphire color, B-flat iron blue, \#F major, gray, green, $\mathrm{bD}$ major dim, bA major dark purple, F major green. In research on music tonal and color, Scriabin found that colors changing from red to violet along with sharps increasing. The Coincident changing of music and color reflects that people's subjective feeling also shows some objective regularity ${ }^{[6]}$ 。 


\subsection{Music Chords Spectrum Analysis}

Because of the diversity of music, there are thousands of chords totally for all kinds of music. But about only twenty tonalities which cover the amount of $90 \%$ of modern music on the basis of statistics. Audio signals from music can be converted into frequency domain, and the spectrum of music can be obtained and displayed ${ }^{[7,8]}$. As standard musical chords, triads of each tonality are analyzed by using Fourier transformation. These standard chords audio files can be obtained through composing software on PC. For audio signals, their characteristics in frequency domain are much more significant than those in time domain. So spectrums of these chords are got by spectrum analysis. Audio signals are random signals which are infinite time domain signals and have no convergence, which does not satisfy the integral condition, then Fourier Transform cannot be performed for these signals directly, so power spectrum analysis is available. By using audio editing software Cool Edit Pro, or the software such as Matlab, the power spectrum analysis on chord music is carried out, the chords spectrum are got, and the spectrum data are stored.

The more intensive varying of a signal in the time-domain signal, the more high frequency components it possesses, and vice versa, the less varying for a signal, the more low frequency components it comprises of. Figure. 1 and Figure. 2 correspond to the A major triad in the time domain and frequency domain waveform respectively, Figure. 3 and Figure. 4 correspond to $\mathrm{G}$ major triad. Composer Rimouski believes that colors of music tones are obvious. Scriabin thinks A major is green. He thinks $\mathrm{C}$ major represents red, which gives a person a kind of special strong emotional color. As to the color of each major, Scriabin considers that along with the increase of sharps, the color changing also from red to violet in spectrum, which is correspondent to frequency increasing for both audio signals and lights.

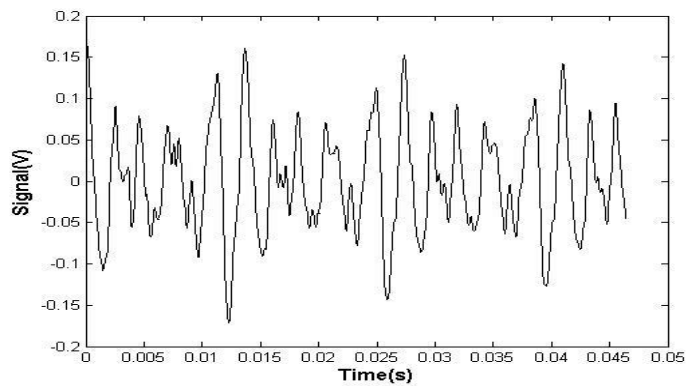

Figure 1. A Major Triad Audio Signal

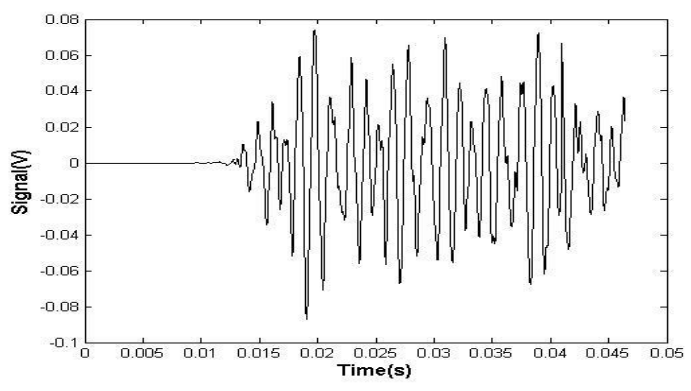

Figure 3. G Major Triad Audio Signal

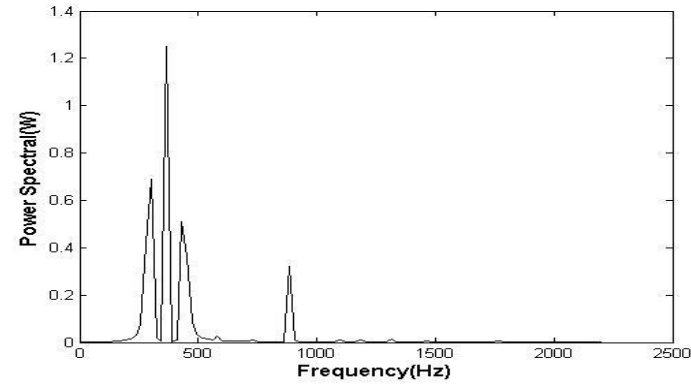

Figure 2. A Major Triad Audio Spectrum

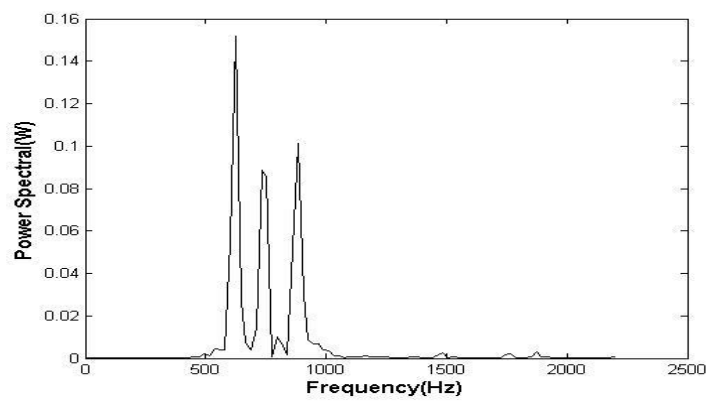

Figure 4. G Major Triad Audio Spectrum 


\subsection{Spectrum Comparison Methods}

The audio signal of music playing is sampled by A/D converter, and the spectrum is obtained by FFT transform[9,10]. In order to implement that music playing matching with color, spectrum analysis only on music playing is still not enough, but the spectrum comparison method must be considered[11]. The most similar chord to music being played at a moment is selected among standard chords through comparing its spectrum to that of music playing, and the color is determined to drive RGB-LED lights. There are several methods for spectrum comparison in which the simplest one is to be determined according to the maximum intensity under a frequency in spectrum, which is the maximum intensity method for the spectrum of contrast signal. The second method is the frequency center of gravity which is based on the spectrum of center of gravity. Also other methods refer to signal correlation analysis, etc. By using Bayesian formula, a probability model of spectrum can be constructed. Spectrum comparison between sampling audio signal and standard music chords can be implemented via statistical calculation. Noises which are generated from the playing audio signal, can seriously affect the accuracy of the audio spectrum identification, Wavelet transform is used to filter high frequency noises[12]. Before performing audio signal processing, procedures of de-noising signal are essential[13]. Wavelet transform adopts analysis window which has fixed area, but with shape changing, to perform signals transform. Wavelet transform has been successfully applied to the audio analysis by its characteristics of multi-resolution analysis.

$\Psi(t) \in L^{2}(R)\left(L^{2}(R)\right.$ stands for square integral real space, that is, limited energy signal space), its Fourier transform is $\Psi(t)$. When $\Psi(t)$ satisfies the following allowed condition,

$$
C_{\psi}=\int_{R} \frac{|\Psi(\Omega)|^{2}}{|\Omega|} d \Omega<\infty
$$

$\Psi(t)$ is a basis function, then

$$
\psi_{a, b}(t)=\frac{1}{\sqrt{a}} \psi\left(\frac{t-b}{a}\right)
$$

Wherein, $a, b$ are constants and $a>0 . a$ is called the scale factor, $b$ is positional parameters, if a, b change constantly, there is a set of functions $\Psi_{a, b}(t)$. The one-dimensional continuous wavelet transformation function is as (3).

$$
W_{f}(a, b)=|a|^{-\frac{1}{2}} \int_{-\infty}^{+\infty} f(t) \psi\left(\frac{t-b}{a}\right) d t
$$

In the application, the wavelet transform selects discrete wavelet transform. Wavelet de-noising methods includes shielding de-noised method, threshold de-noised method, modulus maxima detection de-noised method, etc. The threshold de-noised method, its idea is to use threshold to deal with the transformed lower wavelet coefficients, the wavelet coefficients corresponding to Gaussian noise are set to zero, and those corresponding to signal are kept, then the processing wavelet coefficient reconstructs the original signal to obtain the optimal estimate of the effective signal ${ }^{[14,15]}$. The effect of the wavelet threshold de-noised method is obvious, as shown in Figure. 5. 

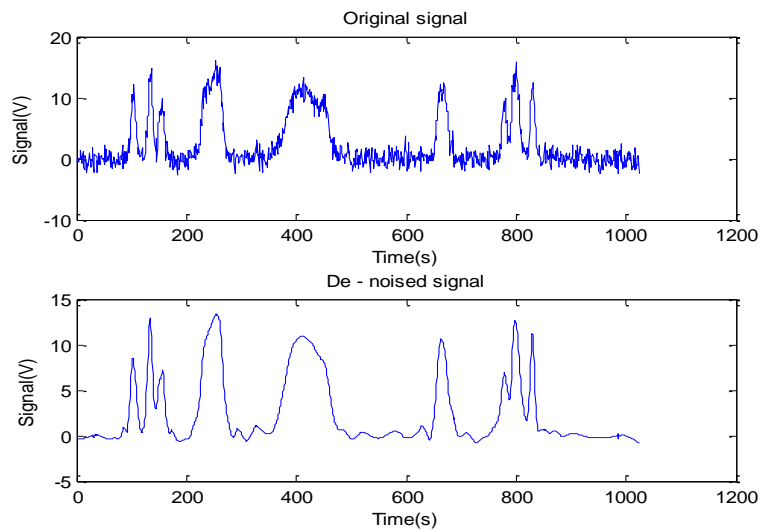

Figure 5. Wavelet De-Noised Threshold Value Method Contrast

\subsection{Analysis on Spectrum Data}

One short piece of chord tone is chosen to be tested. To perform FFT, the frequency of sampling $f_{s}$ is $44 \mathrm{KHz}$ and number of sampling points $N$ is 2048 which is to keep the precision of conversion. The total sampling period last just about $47 \mathrm{~ms}$. According to Nyquist sampling theorem, the highest frequency $f_{m}$ would be $22 \mathrm{KHz}$, and all the audio signals with frequencies in the scope of $f_{m}$ will be differentiated. This can satisfy the application for audible frequency range is about $20 \mathrm{~Hz} 20 \mathrm{KHz}$. A part of the FFT results is shown in Table 1.

\section{Table 1. Data of FFT Results}

\begin{tabular}{c|c|c|c|c|c|c|c|c}
\hline Sequence & 12 & 13 & 14 & 15 & 16 & 17 & 18 & 19 \\
\hline Frequency $(\mathrm{Hz})$ & 236.87 & 258.4 & 279.93 & 301.46 & 323 & 344.53 & 366.06 & 387.6 \\
\hline Amplitude & 0.0314 & 0.0802 & 0.4173 & 0.6889 & 0.0184 & 0.0067 & 1.2495 & 0.0036 \\
\hline Sequence & 20 & 21 & 22 & 41 & 42 & 43 & 44 & 45 \\
\hline Frequency $(\mathrm{Hz})$ & 409.13 & 430.66 & 452.2 & 861.33 & 882.86 & 904.39 & 925.93 & 947.46 \\
\hline Amplitude & 0.0106 & 0.5110 & 0.3634 & 0.0017 & 0.3199 & 0.0089 & 0.0028 & 0.0022 \\
\hline
\end{tabular}

A part of tone scale data which reflects the relations between octaves and frequencies is as shown in Table 2 .

Table 2. Frequencies Corresponding to Tonal Scales

\begin{tabular}{c|c|c|c|c|c|c|c|c|c|c}
\hline Octave & 0 & 1 & 2 & 3 & 4 & 5 & 6 & 7 & 8 & 9 \\
\hline $\mathrm{D}$ & $\begin{array}{c}18.354 \\
(-46)\end{array}$ & $\begin{array}{c}36.708 \\
(-34)\end{array}$ & $\begin{array}{c}73.416 \\
(-22)\end{array}$ & $\begin{array}{c}146.83 \\
(-10)\end{array}$ & $\begin{array}{c}293.66 \\
(+2)\end{array}$ & $\begin{array}{c}587.33 \\
(+14)\end{array}$ & $\begin{array}{c}1174.7 \\
(+26)\end{array}$ & $\begin{array}{c}2349.3 \\
(+38)\end{array}$ & $\begin{array}{c}4698.6 \\
(+50)\end{array}$ & $\begin{array}{c}9397.3 \\
(+62)\end{array}$ \\
\hline $\mathrm{E}$ & $\begin{array}{c}20.602 \\
(-44)\end{array}$ & $\begin{array}{c}41.203 \\
(-32)\end{array}$ & $\begin{array}{c}82.407 \\
(-20)\end{array}$ & $\begin{array}{c}164.81 \\
(-8)\end{array}$ & $\begin{array}{c}329.63 \\
(+4)\end{array}$ & $\begin{array}{c}659.26 \\
(+16)\end{array}$ & $\begin{array}{c}1318.5 \\
(+28)\end{array}$ & $\begin{array}{c}2637.0 \\
(+40)\end{array}$ & $\begin{array}{c}5274.0 \\
(+52)\end{array}$ & $\begin{array}{c}10548 \\
(+64)\end{array}$ \\
\hline $\mathrm{F}$ & $\begin{array}{c}21.827 \\
(-43)\end{array}$ & $\begin{array}{c}43.654 \\
(-31)\end{array}$ & $\begin{array}{c}87.307 \\
(-19)\end{array}$ & $\begin{array}{c}174.61 \\
(-7)\end{array}$ & $\begin{array}{c}349.23 \\
(+5)\end{array}$ & $\begin{array}{c}698.46 \\
(+17)\end{array}$ & $\begin{array}{c}1396.9 \\
(+29)\end{array}$ & $\begin{array}{c}2793.8 \\
(+41)\end{array}$ & $\begin{array}{c}5587.7 \\
(+53)\end{array}$ & $\begin{array}{c}11175 \\
(+65)\end{array}$ \\
\hline $\mathrm{F} \# / \mathrm{Gb}$ & $\begin{array}{c}23.125 \\
(-42)\end{array}$ & $\begin{array}{c}46.249 \\
(-30)\end{array}$ & $\begin{array}{c}92.499 \\
(-18)\end{array}$ & $\begin{array}{c}185.00 \\
(-6)\end{array}$ & $\begin{array}{c}369.99 \\
(+6)\end{array}$ & $\begin{array}{c}739.99 \\
(+18)\end{array}$ & $\begin{array}{c}1480.0 \\
(+30)\end{array}$ & $\begin{array}{c}2960.0 \\
(+42)\end{array}$ & $\begin{array}{c}5919.9 \\
(+54)\end{array}$ & $\begin{array}{c}11840 \\
(+66)\end{array}$ \\
\hline $\mathrm{G}$ & $\begin{array}{c}24.500 \\
(-41)\end{array}$ & $\begin{array}{c}48.999 \\
(-29)\end{array}$ & $\begin{array}{c}97.999 \\
(-17)\end{array}$ & $\begin{array}{c}196.00 \\
(-5)\end{array}$ & $\begin{array}{c}392.00 \\
(+7)\end{array}$ & $\begin{array}{c}783.99 \\
(+19)\end{array}$ & $\begin{array}{c}1568.0 \\
(+31)\end{array}$ & $\begin{array}{c}3136.0 \\
(+43)\end{array}$ & $\begin{array}{c}6271.9 \\
(+55)\end{array}$ & $\begin{array}{c}12544 \\
(+67)\end{array}$ \\
\hline $\mathrm{A}$ & $\begin{array}{c}27.500 \\
(-39)\end{array}$ & $\begin{array}{c}55.000 \\
(-27)\end{array}$ & $\begin{array}{c}110.00 \\
(-15)\end{array}$ & $\begin{array}{c}220.00 \\
(-3)\end{array}$ & $\begin{array}{c}440.00 \\
(+9)\end{array}$ & $\begin{array}{c}880.00 \\
(+21)\end{array}$ & $\begin{array}{c}1760.0 \\
(+33)\end{array}$ & $\begin{array}{c}3520.0 \\
(+45)\end{array}$ & $\begin{array}{c}7040.0 \\
(+57)\end{array}$ & $\begin{array}{c}14080 \\
(+69)\end{array}$ \\
\hline
\end{tabular}


In Table 2, Octave 0 9 represents octave area, and numbers in parentheses represent cents to modify the exactitude frequency to fit scale (1 unit is about $0.25633 \mathrm{~Hz}$ ). In table 1 , the peak points $15,18,21$ and 42 can be found, and even the sequence point 22 is not a peak, but it is adjacent to 21, and its amplitude is also not low. Comparing the frequencies of these peak points to that in Table 2, the corresponding tones to the peak points which are D4, F\#4, A4 and A5 can be determined, and the comparing results are shown in Table 3. In fact, the frequency of A5 is double of that of A4, and it is the resonant frequency to A4. So the component of peak point 42 maybe comes from the original audio signal, or from the electronic resonance. Anyway it is a common phenomenon, not so much severe, and high frequency interference can be easily filtered out. Therefore, the chord tone can be look as the chord A-D-F\# which belongs to A major chord. The chord is corresponding to green color according to Scriabin. When this piece of tone is played, light green color is chosen to match the tone.

Table 3. Frequencies Comparisons

\begin{tabular}{c|c|c|c|c|c}
\hline Peak points & 15 & 18 & 21 & 22 & 42 \\
\hline Frequency $(\mathrm{Hz})$ & 301.46 & 366.06 & 430.66 & 452.2 & 882.86 \\
\hline Amplitude & 0.6889 & 1.2495 & 0.5110 & 0.3634 & 0.3199 \\
\hline Tone & $\mathrm{D} 4$ & $\mathrm{~F} \# 4$ & $\mathrm{~A} 4$ & $\mathrm{~A} 4$ & $\mathrm{~A} 5$ \\
\hline Frequency $(\mathrm{Hz})$ & 293.66 & 369.99 & 440 & 440 & 880 \\
\hline Relative Error & $+2.656 \%$ & $-1.062 \%$ & $-2.122 \%$ & $+2.772 \%$ & $+0.325 \%$ \\
\hline
\end{tabular}

\section{Design of Experimental System Platform}

\subsection{Overall Design}

The experimental platform is a system with a microprocessor embedded. The microprocessor is STC15W4K61S4 with more than 4K ram which has enough capacity as a data buffer for audio file operation. Because audio files require large capacity storage, $\mathrm{SD} / \mathrm{TF}$ cards and $\mathrm{U}$ disks are used. A microprocessor interfaces with a SD/TF card via SPI bus ${ }^{[16,17]}$. U disk data also plays a role in commonality of USB protocol, so that the U disk realizes the portable features, which is more suitable for audio file storage. $\mathrm{CH} 375$ is a general USB bus interface chip, which supports USB host and slave model. CH375 can easily hook up to system bus of MCU/DSP/MCU/MPU controllers ${ }^{[18]}$. Audio files in $\mathrm{SD} / \mathrm{TF}$ cards are accessed by SPI bus, while in U disks can be read via $\mathrm{CH} 375$, then are sent to audio decoder VS1003 which is a single piece of MP3/WMA format audio decoder and ADPCM encoder ${ }^{[19,20]}$. An instrument with a microprocessor embedded is portable, but also heterogeneous a PC ${ }^{[21]}$. Some software such as file system FAT32 that support $\mathrm{SD} / \mathrm{TF}$ card operation should be transplanted to the system. The system overall diagram is shown as in Figure. 6.

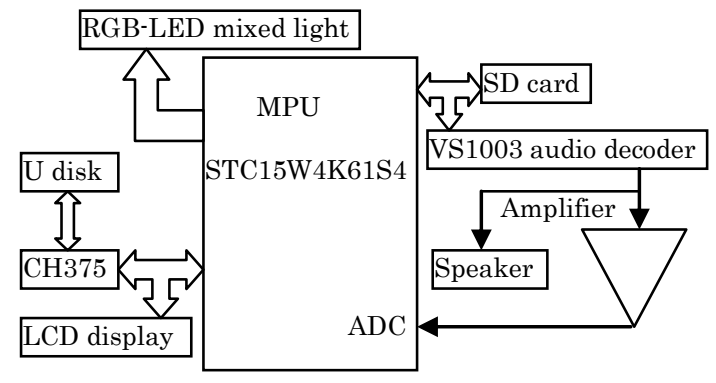

Figure 6. System Overall Diagram 


\subsection{RGB-LED Driver Design}

Red, Green, Blue(RGB) correspond to the three primary colors of light, frequencies of the different colors of LED tubes are different, while they are mixed together in proportion, other else colors are produced ${ }^{[22]}$. International Commission on Illumination (CIE) regards each primary color luminance values as a unit, red, green, three primary colors of light produce white light with equal mixing ratio ${ }^{[23]}$, that is, $(\mathrm{R})+(\mathrm{G})+(\mathrm{B})=$ (W). Similarly, the yellow light is obtained by mixing red and green light, namely, $(\mathrm{R})+$ $(\mathrm{G})=(\mathrm{Y})$; Red and blue lights product magenta, that is, $(\mathrm{R})+(\mathrm{B})=(\mathrm{M})$, and so on. If unequal proportions of lights are mixed, more colorful lights are obtained. All kinds of mixed lights are implemented by using three PWM outputs that driving RGB-LED lights. Duty ratios (T1/T) determine mixed RGB-LED light effects, PWM driving waveforms are as shown in Figure. 7.

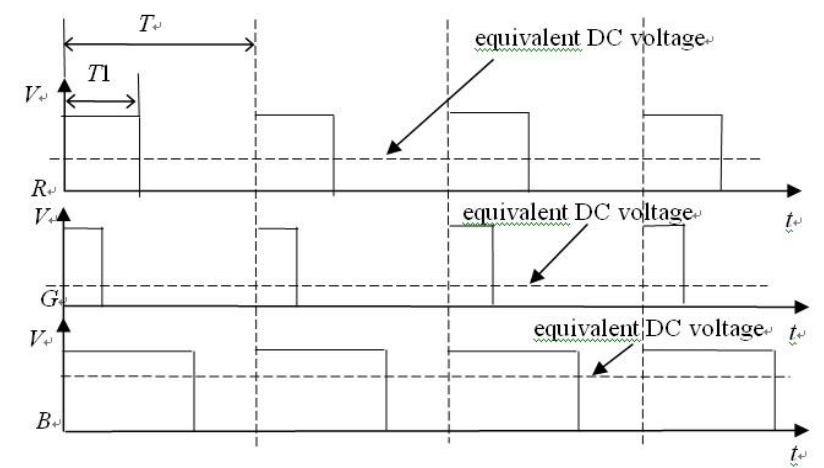

Figure 7. RGB-LED Driving Waveform via Three PWM Outputs

\section{Experimental Tests}

On the experimental platform, audio files are stored in the SD/TF card, or U disk, the audio data should be accessed in file form via MPU STC15W4K61S4. As to SD/TF card, file system ZnFAT which is compatible with FAT32 is adopted in the embedded system. As an interface chip of a $\mathrm{U}$ disk, $\mathrm{CH} 375$ contains a library of functions that support file operations. When a $\mathrm{U}$ disk is inserted, function CH375DiskReady() determines that the $\mathrm{U}$ disk is ready, and then the capacity of the $\mathrm{U}$ is checked throughCH375DiskSize(), and the information such as files on the $U$ disk is obtained afterwards. The MPU must read U disk and then output the data to the audio decoder VS1003 continuously to keep audio data stream to make music playing. The U disk information is read by the MPU as shown in Figure. 8. 


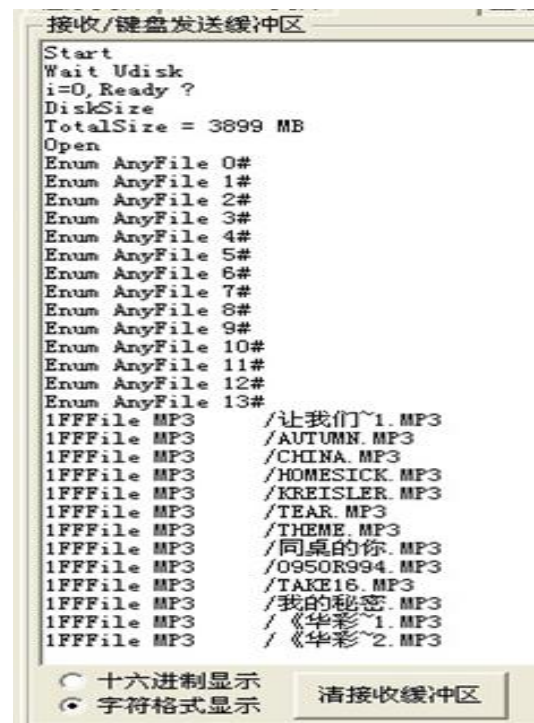

Figure 8. Information of U Disk
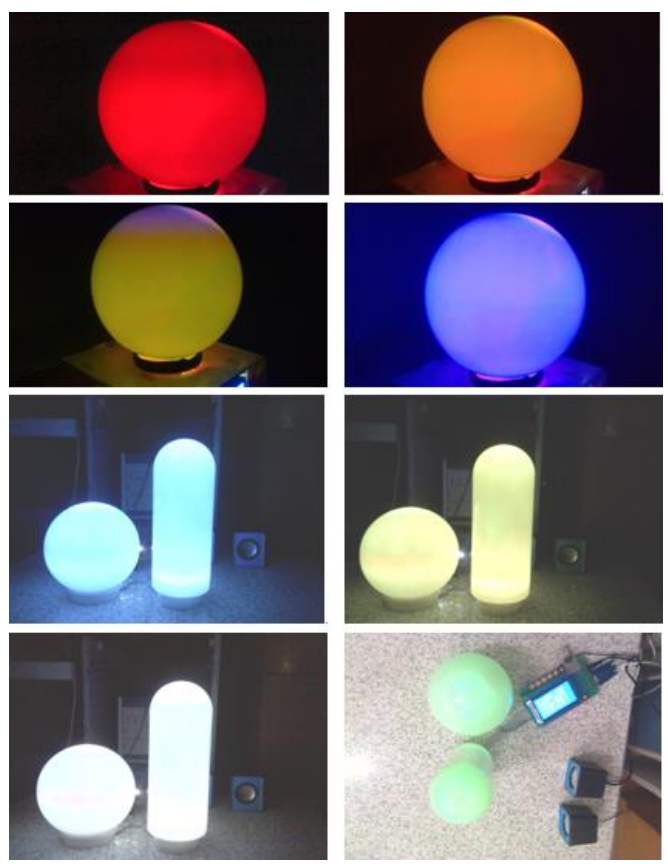

Figure 9. RGB - LED Mixed Light and Playing Music Matching Test

Audio signals of playing music are sampled. The sampling frequency conforms to Nyquist sampling theorem, and sampling number is decided by the precision of spectrum analysis. The interval between two groups of sampling data is arranged in 200 to 500 milliseconds. FFT transformation is executed accordingly to get the spectrum of audio signals in real time. Through experiments, all kinds of software functions such as accessing SD/TF card and U disk, VS1003 operating, audio signal sampling, FFT transforming and processing, RGB-LED driving, displaying and keyboard processing etc. are implemented which are scheduled to be arranged in a correct timing sequence. From tests, purposes to optimize the audio signal processing parameters, evaluate the efficiency of audio frequency spectrum identification, drive RGB-LED mixed lights via PWM outputs are reached. A color is determined corresponding to the music at a moment and 
the effect is implemented that matching colors are presented automatically to arbitrary playing music. Patterns of RGB-LED lights mixed matching music playing are shown in Figure. 9.

\section{Conclusions}

Although there are many studies on the relationship between music and color, most of them are still in phases of theoretical analysis. There are some devices with music playing accompanied by light color flashing. But often melodies are fixed, and patterns of light color are pre arranged, or light just flashes randomly without matching between music and color. Atherton develops a novel music player with which RGB-LED lights are controlled by microprocessor LumiGeek. Atherton purpose is to implement the effect that light color changing is along with music playing. But his research work is still not succeeded (http://www.narkii.com/news/news_98059.shtml). Comparing to these products, our research possesses obvious advantage based on audio spectrum analysis and recognition. This study involves techniques of audio files playing, audio signal sampling, spectrum analysis on audio signals, and RGB-LED driving etc. It is impossible to determine colors that corresponding to music playing only by audio signals spectrums of music being played. Through comparison methods, considering spectrums of music chords and that of audio signals together, spectrum identification of the similar chord to the playing audio signal determines the corresponding color of the playing music. Music chords must be used as standard references. Through comparisons among spectrum of music chords and audio signals being played, the most similar chord to music playing is selected, and the corresponding color is determined, then RGB-LED lights are driven by PWM outputs. Wavelet transform is used to effectively eliminate high frequency interferences to enhance the accuracy of spectrum identification. Through experimental testing, it reaches the effect that RGB-LED mixed lights match well with music playing.

\section{Acknowledgment}

This work is supported by Project of Natural Science Foundation of China (NSFC 53177037), Project of Education Department of Heilongjiang Province (12541177), and Heilongjiang Students Innovative Experiment Project(201410214036).

\section{References}

[1] P G Grossenbacher, C T Lovelace, "Mechanisms of synaesthesia: Cognitive and physiological constraints", Journal of Trends in cognitive sciences, vol. 5, no.1, (2001), pp.36-41.

[2] E Schubert, "The influence of emotion, locus of emotion and familiarity upon preference in music", Journal of Psychology of Music, vol.35, no.3, (2007), pp.499-515.

[3] J Simner, C Mulvenna, N Sagiv, "Synaesthesia: The prevalence of atypical cross-modal experiences", Journal of PERCEPTION-LONDON, vol.35, no.8, (2006), pp.1024-1033.

[4] T Tsang, K B Schloss, "Associations between Color and Music are Mediated by Emotion and Influenced by Tempo", Journal of The Yale Review of Undergraduate Research in Psychology, pp. 82-93, 2010.

[5] H. Zhang, H. B. Wang, G. Y. Lin, et al. Design and Implementation of a Device with RGB Driving Based on FFT, ICIC Express Letters Part B: Applications, vol.5, no.4, (2014), pp.1027-1032,

[6] Jeff. Music and color, Musical Instrument[J], vol.9, pp.52-53, 2002.

[7] J Lin, D Qiu Lei, "The systematic study of music spectrum display based on FFT", Advanced Materials Research, vol. 945, (2014), pp.1764-1767.

[8] S Wei, G Longbao, C Long, "New solution to signal generator and distortion analyzer", Chinese Journal of Scientific Instrument, vol.30, no. 11, (2009), pp.2249-2254.

[9] H. Zhang, G. Y. Lin, "Design and implementation of mixed light effect based on the audio signal frequency spectrum analysi"s, Proc. of 8th International Forum on Strategic Technology 2013, IFOST 2013. Ulaanbaatar, Mongolia, (2013), pp.68-74.

[10] H. Zhang, C. W. Tian, G. Y. Lin, et al. "Design and implementation of mixed light effect based on the audio signal spectrum analysis, Journal of Harbing university of science and Technology," vol.18, 
no.6, (2013), pp.47-51.

[11] Y. C. Fu, H. Zhang, G. Y. Lin, et al. "Research and Implementation of RGB-LED Mixed Light Matching along with Music Playing”, Journal of Harbing university of science and Technology, vol.19, no.6, (2014), pp.48-53.

[12] A. C. Pozo, R. T. Codorniu, "Comparative review of denoising techniques for industrial signals using Discrete Wavelet Transform and adaptive threshold selection", Journal of RIAI-Revista Iberoamericana de Automatica Informatica Industrial, vol. 10, no.2, (2013), pp.143-148.

[13] W Fang, J Zhong, P Chenglin, "Research on ECG signal denoising based on dual-tree complex wavelet transform", Chinese Journal of Scientific Instrument, vol.34, no. 5, (2013), pp.1160-1166.

[14] H. Yuqing, W. Youren, et al. New signal de-noising method based on fractional wavelet packet transform in time-frequency domain, Chinese Journal of Scientific Instrument, vol. 32, no.7, (2011), pp.1534-1539.

[15] Y Zhenzhen, Y Zhen, "Adaptive conjudate gradient projection algorithm for noisy speech compression and reconstruction”, Chinese Journal of Scientific Instrument, vol.33, no. 10, (2012), pp.2200-2207.

[16] M. Stojan, A. Zoran, Digital sound recorder with ARM microcontroller and SD card, Proc. of 20th Telecommunications Forum, TELFOR 2012. Belgrade, Serbia, (2012), pp.1741-1743.

[17] D Xueming, L Qiaowei, Y Dongsong, Z Yifei, and F Kanjun, "A Design of Automatic Bus Station Reporter Based on VS1003 and SD card". Proc. of 2013 International Conference on Structures and Building Materials, Guizhou, China, (2013), pp.2847-2850.

[18] D. Z. Sun, "Design and implementation of MP3 player based on FPGA[C]". Applied Mechanics and Materials , vol. 443, (2014) pp.746-749.

[19] H. Zhang, C. W. Tian, G. Y. Lin, Implementation of a wireless controlled device with RGB driving based on FFT, Journal of International Journal of Multimedia and Ubiquitous Engineering, vol.8, no.6, (2013), pp.151-158

[20] A. L. Zoranovic, G. M. Stojanovic, V. D. Malbaša, "Development of an MP3 player using an MP3 decoder", Journal of International Journal of Electrical Engineering Education, vol.47, no.3, (2010), pp.329-342.

[21] H Yongfu, W Shaojun, P Yu, "High performance heterogeneous embedded computing: a review.", Instrumentation, vol. 1, no. 2, (2014), pp.1-12.

[22] O. Minoru, S. Tomoyoshi, Y. Takuto, Time-division color electroholography using one-chip RGB-LED and synchronizing controller, Journal of Optics Express, vol.19, no.13, (2011, pp.12008-12013.

[23] G. S. Chang, Y. H. Jong, S. G. Jin, "Improvement of Color and Luminance Uniformity of the Edge-Lit Backlight Using the RGB-LEDs", Journal of the Optical Society of Korea, vol.15, no.3, (2011), pp.272-277. 